\title{
Potencial efeito preventivo e terapêutico da actividade física no síndrome do canal cárpico
}

\author{
Vasco Neves ${ }^{1}$ \\ José Duarte ${ }^{2}$
}

https://doi.org/10.5628/rpcd.05.01.114

\author{
${ }^{1}$ Escola Secundária Condes de Resende, Canelas, Portugal \\ ${ }^{2}$ Universidade do Porto, Faculdade de Ciências do Desporto \\ e de Educação Física, Portugal
}

\section{RESUMO}

O síndrome do canal cárpico (SCC) é a doença de sobrecarga relacionada com a actividade laboral que surge mais frequentemente, tendo a sua incidência aumentado exponencialmente nos últimos vinte anos (28). Os seus sintomas, atingindo pessoas com variadas ocupações e profissões, são extremamente limitativos para o indivíduo afectado, influenciando negativamente a sua vida profissional e pessoal (117). Por outro lado, o tratamento deste distúrbio musculo-esquelético é, frequentemente, moroso, oneroso e incompatível com as obrigações profissionais (27). Assim, a prevenção do SCC ocupa um lugar de destaque no combate a esta doença, podendo evitar todos os inconvenientes a ela associados. O objectivo deste trabalho consiste em efectuar uma revisão bibliográfica acerca do SCC e obter dados que permitam esclarecer o papel da actividade física (AF) na sua prevenção primária e secundária. Desse modo, foram revistos os principais factores de risco para o aparecimento e desenvolvimento do SCC (destacando-se, entre outros, o elevado índice de massa corporal - IMC -, a idade acima dos 30 anos, as actividades manuais repetitivas e a diabetes mellitus - DM) e as principais intervenções preventivas, primárias e secundárias, sugeridas pela literatura. Verificou-se que as principais medidas preventivas são baseadas na minimização ou na anulação dos factores de risco da doença e que a AF foi pouco investigada enquanto potencial estratégia preventiva do SCC. Os estudos efectuados com o intuito de esclarecer a relação entre a AF e a prevenção do SCC são muito reduzidos e foram, maioritariamente, desenvolvidos recorrendo a amostras pouco numerosas. Contudo, numa revisão bibliográfica mais ampla, parece encontrar-se motivos que justifiquem, pelo menos, uma investigação mais aprofundada das potencialidades da actividade física na prevenção do SCC.

Palavras-chave: síndrome do canal cárpico, actividade física, factores de risco, prevenção.

\section{ABSTRACT \\ Potential effect of physical activity on therapy and prevention of carpal tunnel syndrome.}

The carpal tunnel syndrome (CTS) is the most frequent work-related overuse disease, having its incidence increased exponentially in the last twenty years (28). The illness symptoms, reaching people with varied occupations and professions, are extremely limitative with a negative influence in professional and personal life (117). On the other hand, this musculoskeletal disorder treatment is, frequently, slow, onerous and incompatible with professional obligations (27). Thus, CTS prevention seems to be a priority measure to fight this disease and the associated inconveniences. The main aim of this work consists in accomplish a bibliographical revision concerning CTS and obtain data that allow clarification about the role of physical activity $(P A)$ in its primary and secondary prevention. In this way, the main risk factors for the appearance and development of CTS have been reviewed (being distinguished, among others, the high body mass index - BMI -, the age above 30 years, the repetitive hand activities and the diabetes mellitus - DM) as well as the main preventive interventions, suggested by literature. It was verified that i) the main prevention measures are based on the minimization of the illness risk factors and ii) the PA was little investigated as a preventive potential strategy for the manifestation of CTS. In fact, studies that intended to clarify the relation between PA and the prevention of CTS are very few and have been, in most cases, developed with insufficient samples. However, according to the bibliography, it seems justified a more deepened experimental investigation about the potential effects of PA on CTS prevention.

Key Words: carpal tunnel syndrome, physical activity, risk factors, prevention. 


\section{INTRODUÇÃO}

Alguns problemas médicos estão intimamente relacionados com a ocupação profissional das pessoas. Nesse conjunto de problemas, as lesões crónicas causadas por sobrecargas continuadas nos tecidos musculo-esqueléticos têm grande relevo $(7,27,82,102$, 109). Não são raras as actividades profissionais que obrigam a movimentos repetitivos e de elevada actividade muscular efectuados em posturas anti-anatómicas que se prolongam no tempo. Estas situações acabam por sobrecarregar as estruturas musculoesqueléticas $(7,19,42,51,60,69,73)$ envolvidas no movimento, sujeitando o indivíduo a lesões de carácter crónico, inflamatório e de restabelecimento difícil. A lesão de sobrecarga é uma inflamação não infecciosa (98), causada por um uso excessivo ou inabitual (62) que ultrapassa o limite das possibilidades de adaptação-reparação das estruturas anatómicas (71), resultando em danos permanentes nas estruturas musculo-esqueléticas devido a tensões excessivas agudas ou crónicas (por repetição) nos tendões, nas baínhas sinoviais dos tendões, nos ligamentos ou nos músculos (98).

Qualquer que seja a actividade profissional relacionada com o aparecimento deste tipo de lesão, o indivíduo atingido padecerá de sintomas que o inibirão de desenvolver a sua actividade profissional com o rendimento habitual. Para além do decréscimo de rendimento, o próprio bem-estar da pessoa afectada poderá ser seriamente comprometido $(7,22,27$, 109). A estas consequências, acrescenta-se o facto de o indivíduo ser obrigado a interromper ou a reduzir a sua actividade e a iniciar um tratamento médico para procurar restabelecer a eficácia habitual anterior à lesão na realização da sua actividade ocupacional e para repor os seus níveis de conforto e de bem-estar. Esse tratamento é, muitas vezes, moroso, oneroso e incompatível com as exigências profissionais $(7,27$, $32,62,97)$. Desse modo, há todo o interesse em prevenir o aparecimento dos distúrbios musculoesqueléticos relacionados com a actividade profissional, de forma a evitar as consequências anteriormente referidas.

A região corporal mais afectada pelas lesões de sobrecarga em diferentes ocupações e profissões é o membro superior $(22,27,82,109)$. A incidência de lesões nesse segmento corporal, associadas a movi- mentos repetitivos, aumentou rapidamente nos derradeiros 20 anos $(19,22,27)$. Assim, é com naturalidade que nos últimos anos tem havido um interesse acrescido na prevenção, no diagnóstico e no tratamento da dor no membro superior (MS), causada por fenómenos de sobrecarga dessa estrutura anatómica $(37,102)$.

As zonas do MS mais afectadas por lesões de sobrecarga são a mão e o pulso (7). De acordo com numerosos estudos efectuados dentro das várias tecnopatias relacionadas com o trabalho, o síndrome do canal cárpico (SCC) é a que surge com maior frequência $(32,44,87,94,123)$. De facto, o SCC atinge indivíduos com variadas ocupações e profissões que têm em comum a execução de movimentos repetitivos da mão e do pulso para a consecução das tarefas laborais. Assim, por exemplo, profissões tão diferentes como operários não especializados (69), técnicos administrativos que utilizam constantemente o teclado ou o rato do computador (101), especialistas em linguagem gestual (87), músicos instrumentistas (62) ou desportistas de alto rendimento $(71,97)$, podem ser alvo desta doença.

O indivíduo atingido pelo SCC será vitimado por uma constelação de sintomas (e.g. diminuição da força muscular, perda de agilidade - diminuição da velocidade nos movimentos -, diminuição da precisão dos movimentos, dor local ou difusa na mão, edema, entorpecimento, atrofia muscular e diferentes fenómenos vasomotores) que, tal como em qualquer lesão de sobrecarga, o prejudicará de forma significativa na sua vida profissional e privada $(62,82)$. Além das consequências directas causadas pela patologia adjacente à doença, o facto da base do tratamento de qualquer lesão de sobrecarga ser o repouso $(27,71,97)$ implica que o paciente tenha que reduzir drasticamente o número de horas de trabalho por dia ou mesmo interromper totalmente as suas actividades, o que poderá ser extremamente prejudicial em algumas profissões ou ocupações onde os resultados finais dependem das horas de prática ou de treino (como por exemplo, em atletas de alto rendimento que têm um calendário desportivo a cumprir ou em músicos instrumentistas, que têm concertos, recitais ou audições agendadas). Desta forma, em qualquer profissão onde o indivíduo esteja dependente da qualidade dos movimentos 
executados pelo seu corpo - mais concretamente, pelos seus MS -, é vital a prevenção para impedir o aparecimento do incapacitante SCC. Assim, este trabalho procura explorar a origem e as formas de prevenção desta frequente lesão de sobrecarga.

Deste modo, o objectivo deste trabalho consiste em efectuar uma revisão bibliográfica acerca do SCC, analisando a sua etiologia, fisiopatologia e os diferentes factores de risco, e verificar se a AF é, de facto, um agente importante para a sua prevenção primária e secundária. Este objectivo é sustentado pela ideia de que a AF, devidamente controlada e adaptada à situação em questão, é uma forma segura de prevenir o aparecimento de lesões no aparelho locomotor $(5,71,97,112)$.

\section{ETIOLOGIA E FISIOPATOLOGIA}

Nos últimos anos, face ao aumento das lesões de sobrecarga relacionadas com o trabalho $(7,27,109)$ e à inflação dos custos inerentes à proliferação desse tipo de lesões (27), tem havido um interesse acrescido na prevenção, no diagnóstico precoce e no tratamento de doenças causadas por fenómenos de sobrecarga (102). Assim, vários autores têm-se debruçado sobre a sua etiologia e fisiopatologia, de forma a possibilitar uma prevenção mais sólida e um tratamento mais eficaz.

De todas as lesões de sobrecarga, o SCC é considerado, por vários autores, a tecnopatia relacionada com o trabalho que surge mais frequentemente $(32,44$, $87,94,103,123)$. O canal cárpico localiza-se na região anterior do pulso e consiste numa goteira (goteira do carpo) que é fechada anteriormente pelo ligamento anular do carpo (64). Esse canal é delimitado, exteriormente, pelos tubérculos do escafóide e do trapézio e, interiormente, pelo osso pisiforme e pela apófise unciforme (do osso unciforme), servindo de passagem para o nervo mediano, para os tendões flexores dos dedos e para o tendão do grande palmar (43). Através desta descrição é possível compreender que o canal cárpico é uma estrutura pouco elástica por onde passam tecidos moles. Esses tecidos movimentam-se com a mudança de posição do pulso e da mão, estando sujeitos a forças de atrito $(11,22,98)$.

Deste modo, é compreensível que o estreitamento do canal cárpico e/ou o aumento do volume dos tecidos moles que o ocupam $(11,80,104)$, promovam uma subida dos valores de pressão no seu interior. Esse aumento de pressão é apontado por numerosos autores como um elemento importante para a patogénese do SCC $(7,11,49,50,89,92,107,117)$. Os valores de pressão mais elevados sujeitam o nervo mediano a superiores forças de fricção e de compressão $(7,35,117)$, podendo originar o SCC que, segundo Werner e Andary (117), é uma constelação de sintomas associados à compressão deste nervo no pulso.

Os movimentos do pulso parecem ter uma elevada repercussão no aumento da pressão interna do canal cárpico (PCC). Assim, as posições de flexão extrema, particularmente se somadas a movimentos não neutrais do antebraço - quando este se encontra em pronação ou supinação -, contribuem para aumentar a PCC $(20,50)$. Werner e Armstrong (118) compartilham a opinião de que as posições do pulso influenciam de forma dramática a pressão hidrostática do canal cárpico, porém afirmam que os movimentos de extensão têm um contributo muito mais importante para o seu aumento do que os movimentos de flexão.

Para além das posições a que o pulso está sujeito, a repetição do movimento é outro factor condicionante da PCC $(117,118)$. A actividade repetitiva da mão pode causar o progressivo espessamento das baínhas sinoviais dos tendões que partilham o canal cárpico com o nervo mediano, aumentando o volume de tecidos moles e inflaccionando a pressão mecânica no interior do canal cárpico (117).

Tendo em consideração que as posturas extremas do pulso e a repetição dos movimentos efectuados por essa articulação influenciam a PCC, facilmente se pode compreender porque é que profissões tão variadas como operários não especializados (69), técnicos administrativos que utilizam constantemente o teclado ou o rato do computador (101), especialistas em linguagem gestual (87) ou músicos instrumentistas (62), são alvos preferenciais do SCC. De facto, todas estas profissões têm em comum a necessidade do indivíduo recorrer a movimentos repetitivos do pulso, sendo frequentemente utilizadas posições extremas de flexão e de extensão da mão que promovem um aumento da PCC, estimulando a patogénese do SCC. 
Outro factor que parece influenciar a PCC é a flexão dos dedos (para refs. ver 31). Segundo Werner et al. (119) essa pressão é mínima quando a mão está relaxada, com os dedos flectidos a 30 graus.

$\mathrm{O}$ aumento da PCC, para além de estar directamente relacionado com o aparecimento do SCC $(7,49,50$, $89,107,117)$, pode resultar em danos isquémicos nos tendões e no nervo mediano que passam pelo canal (7). Assim, o aumento da PCC acaba por promover o aparecimento de outro factor de risco para o desenvolvimento do SCC: a isquemia aguda ou crónica do nervo mediano $(28,116)$. Desta forma, o nervo mediano poderá ser danificado, simultaneamente, por um aumento da PCC e/ou pelos efeitos da isquemia (116).

\section{FACTORES DE RISCO}

Na literatura está descrita uma abundância de factores de risco para o SCC, porém não existem informações precisas acerca do nível de exposição em que qualquer um desses factores começa a ter um efeito significativo para o aparecimento ou evolução da doença (28). Por outro lado, tendo sido utilizados vários métodos para descobrir e identificar esses factores de risco (para refs. ver $8,28,61,66$ ) existe alguma dificuldade em classificá-los, uma vez que alguns dados são contraditórios, o que constrange o estudo da relação factor de risco/SCC $(7,8,28,116)$. Apesar de na literatura existirem diferentes formas de classificação dos factores de risco, neste trabalho eles serão agrupados em quatro grupos fundamentais: factores ocupacionais, factores biológicos, factores psicológicos e factores clínicos (onde serão incluídos os antecedentes médicos).

\section{Factores ocupacionais}

Apesar de alguns autores questionarem o facto do SCC estar relacionado com a profissão exercida (40, 76), há fortes evidências que suportam uma relação directa entre o aparecimento dessa doença e os factores ocupacionais $(3,65,95)$. Assim, embora existam poucos estudos epidemiológicos acerca dos factores ocupacionais que contribuem para o desenvolvimento desse síndrome de sobrecarga (27), foi identificada uma forte correlação entre a actividade manual e o SCC $(2,3,25,55,78,93)$.

Desse modo, a repetitividade da tarefa, a utilização de posturas anti-anatómicas e a realização de tarefas pesadas que exijam uma força de preensão elevada, parecem ser factores ocupacionais unanimemente aceites como sendo de risco, no desempenho de tarefas manuais, para o desenvolvimento da doença $(28,55,82,91)$. A estes factores, alguns autores acrescentam ainda a compressão mecânica localizada, a vibração a que a mão e o pulso estão sujeitos (normalmente transmitida pela ferramenta utilizada no desempenho das tarefas profissionais) e as reduzidas temperaturas ambientais existentes no local de trabalho $(28,82,111)$.

Apesar de qualquer um dos factores enunciados ser definido como de risco para o aparecimento do SCC, na realidade é, fundamentalmente, o efeito somatório e combinado de todos eles que condiciona o desenvolvimento da doença $(41,82)$. Corroborando esta opinião, Nelson et al. (77) afirmam que quando dois factores de risco estão simultaneamente presentes, há um efeito de sinergia que provoca mais danos do que a simples soma dos dois. Relativamente a esta interacção de factores, Macfarlane (66) salienta o facto de haver evidências que apontam para a existência de uma relação forte entre o trabalho altamente repetitivo, com elevadas solicitações ao nível da força de preensão e efectuado em posições antianatómicas, e o aparecimento do distúrbio musculoesquelético em questão. Para demonstrar a importância que a conjugação dos diferentes factores de risco parece ter no desenvolvimento da lesão de sobrecarga analisada, é importante destacar a opinião de Downs (27), que considera não existirem evidências suficientes que permitam acreditar que o simples aparecimento de um dos factores de risco (enunciados anteriormente) aumente as possibilidades de desenvolvimento de lesões de sobrecarga tais como o SCC, acrescentando que esses factores ainda não foram estabelecidos como acontecimentos causais de condições patológicas específicas.

\section{Factores biológicos}

Segundo diversos autores, os factores biológicos que estão relacionados com uma maior probabilidade do indivíduo desenvolver o SCC são o sexo feminino, um elevado Índice de Massa Corporal (IMC) e a idade acima dos 30 anos $(11,28,56,66,75,78,99$, $105,111,119)$. 
Relativamente ao sexo do indivíduo, é incontestável a existência de uma maior predisposição do sexo feminino para o desenvolvimento desta doença, sendo numerosos os estudos que consideram o sexo do indivíduo como um factor de risco independente e determinante para o aparecimento do SCC $(8,11$, $66,119)$. Além disso, um estudo efectuado por Becker et al. (8) concluiu que esse factor de risco parece ser o único factor pessoal correlacionado, de forma positiva, com a severidade da doença; ou seja, os doentes do sexo feminino apresentam uma tendência superior para desenvolverem casos mais severos de SCC.

Uma das justificações possíveis para o facto das mulheres serem mais frequentemente vitimadas pelo SCC está relacionada com as suas características antropométricas, significativamente diferentes das dos homens (28). Assim, essas diferenças, tais como as circunferências do pulso ou o tamanho do osso radial, podem ser a causa de diferentes índices de flexibilidade nos dois sexos (70), que permitem a adopção de posturas mais extremas (frequentemente anti-anatómicas) por parte das mulheres, o que, ao elevar a PCC, se constitui como um factor de risco para o desenvolvimento do SCC (28).

Nos estudos efectuados por Werner et al. (119) e Stallings et al. (99), um elevado IMC (IMC >30) também revelou ser um factor de risco independente e estatisticamente significativo quando analisado separadamente. Segundo Becker et al. (8), a relação entre a obesidade e o SCC pode ser explicada pela acumulação de tecido gordo dentro do canal cárpico, situação que aumenta a pressão hidrostática nesse canal e exerce um efeito compressivo sobre o nervo mediano.

Embora a idade seja considerada um factor de risco importante, não foi possível encontrar, entre os diversos estudos, um valor de referência consensual que correspondesse a uma faixa etária de risco acrescido para o aparecimento e desenvolvimento do SCC. Contudo, a maioria dos autores concorda com o facto de que o aumento das probabilidades de desenvolver o SCC aumenta depois de se atingirem os 30 anos de idade $(54,99,111,119)$.

Para além destes três factores biológicos de risco, a literatura apresenta outros que, sem serem tão valorizados como os que foram referidos, parecem estar relacionados com o surgimento ou a gravidade do distúrbio musculo-esquelético em questão. Dentro desses factores é importante destacar a raça branca, a hipermobilidade articular pré-existente, a entrada na menopausa e a baixa aptidão física $(28,53,66$, 78 ), aos quais Lange (58) acrescenta a variabilidade anatómica do músculo palmaris profundus.

\section{Factores psicológicos}

Contrastando com os factores ocupacionais, a influência dos factores psicológicos no aparecimento e desenvolvimento do SCC tem recebido uma atenção reduzida (66). Contudo, esses factores parecem ser importantes quer na progressão dos sintomas da doença, quer no próprio surgimento da mesma (12, $27,44,66,109)$. O stress percebido, a reduzida satisfação no trabalho, a pressão causada pela obrigação de cumprir tarefas num limitado espaço de tempo, a falta de apoio social e um trabalho monótono parecem ser alguns factores associados ao desenvolvimento de sintomas musculo-esqueléticos associados às lesões musculo-esqueléticas de sobrecarga (12, 44). Um estudo de grande escala efectuado em França (59) permitiu concluir que os indivíduos com reduzido bem-estar psicológico, que tinham um diminuto controlo sob o seu trabalho e que eram constantemente pressionados pelo dever de cumprir prazos, estavam mais predispostos a desenvolverem esse tipo de sintomas. Além deste estudo, outros foram efectuados concluindo que os factores psicológicos são importantes no aparecimento e na definição da severidade do SCC (66).

\section{Factores clínicos}

São vários os antecedentes médicos que podem influenciar e fomentar o aparecimento do SCC (28). Dentro desses antecedentes, alguns dos mais citados são os distúrbios prévios musculo-esqueléticos, as cirurgias ginecológicas, as varizes, os antecedentes de fracturas de pulso, os tratamentos de emagrecimento e um elevado número de doenças sistémicas, tais como os diabetes mellitus (DM), a artrite reumatóide e o hipotiroidismo $(21,52,53,81,119)$. Porém, dentro destes factores clínicos, aquele que foi mais estudado e, simultaneamente, o que parece estar mais relacionado com o desenvolvimento do SCC é a DM $(8,80)$. De facto, alguns dos estudos 
mais recentes que investigaram a relação entre a DM e esta doença revelaram que esse factor de risco está relacionado de forma estatisticamente significativa com o aparecimento do SCC $(8,80)$. Contudo, tal como afirmam Becker et al. (8), a DM está frequentemente associada com a obesidade, o que poderá causar alguma interferência nas conclusões registadas. Tendo em conta que, por um lado, não foi encontrado nenhum estudo que analisasse profundamente essa fonte de erro e que, por outro lado, alguns estudos efectuados não conseguiram provar a relação entre a DM e o desenvolvimento do SCC (26), deve-se manter em aberto, tal como afirmam Becker et al. (8), a possibilidade da DM não ser um real factor de risco, estando apenas associada ao SCC devido à sua íntima ligação com um outro forte factor de risco: a obesidade.

Apesar de, tal como já referido, ainda existir alguma confusão entre estas variáveis (factores de risco), bem como acerca dos efeitos das diferentes associações - fundamentais no desenvolvimento do SCC que podem ocorrer entre cada uma delas (8), os factores de risco mais consistentes com os estudos efectuados são o género feminino, a obesidade, um elevado índice de massa corporal (IMC), a idade acima dos 30 anos, as actividades motoras repetitivas e a DM $(53,54,78,99,105,111,119)$.

Contudo, é importante salientar que estes dados, provavelmente, reflectem a tendência que a literatura especializada tem revelado em debruçar-se particularmente sobre os factores ocupacionais e biológicos, havendo ainda reduzidos estudos acerca dos outros factores apresentados, o que se pode traduzir numa subvalorização dos mesmos (66).

\section{MANIFESTAÇÕES CLÍNICAS}

Quando uma doença de sobrecarga das estruturas musculo-esqueléticas se instala, o seu tratamento torna-se a única forma de restabelecer a eficácia habitual na realização da actividade ocupacional e de repor os níveis de conforto e de bem-estar anteriores à sua consolidação. Esses tratamentos, para além de serem, muitas vezes, incompatíveis com as exigências profissionais $(7,27,32,62,97)$, segundo Weber (114) são, na sua maioria, ineficazes e demonstram falta de bases científicas - apesar dos pacientes com lesões de sobrecarga terem ao seu alcance uma ele- vada variedade de tratamentos (desde a fisioterapia até à medicina alternativa), nenhum deles é baseado em evidências científicas sólidas. Desse modo, há todo o interesse em prevenir o aparecimento dos distúrbios musculo-esqueléticos relacionados com a actividade profissional, de forma a evitar as consequências anteriormente referidas.

Embora as profissões e ocupações que parecem promover o aparecimento do SCC sejam variadas, os sintomas que afectam as vítimas dessa doença são semelhantes, prejudicando, de forma directa, as suas vidas profissionais e privadas $(7,22,27,109)$. Os sintomas mais frequentemente detectados e descritos pela literatura são a redução da capacidade de produzir intensidades elevadas de força de preensão (devido à diminuição da capacidade dos músculos tenares - inervados pelo nervo mediano - em produzirem força), dor e parestesia nas mãos, diminuição da precisão nas habilidades que requerem a capacidade de preensão, diminuição da sensibilidade nas zonas de distribuição do nervo mediano (polegar, indicador, médio e bordo lateral externo do anular) e do pulso $(9,38,45,48,116,117)$. Apesar de, classicamente, se considerar que a maioria dos sintomas descritos se localizam na distribuição do nervo mediano $(8,11,116)$, estudos sistemáticos sugerem que podem também atingir o antebraço, o cotovelo, o braço, o ombro e o pescoço (para refs. ver 8, 100). Facilmente se pode compreender a influência negativa que todos estes sintomas podem ter na vida de um indivíduo atingido pelo SCC. Desse modo, quando esses sintomas começam a surgir, torna-se imperativo o tratamento desta doença. Infelizmente, tal como na maioria das doenças provocadas por sobrecargas musculo-esqueléticas, esses tratamentos são, frequentemente, morosos, dispendiosos e de difícil articulação com as exigências profissionais (7, 109, 117) - o facto da base do tratamento de qualquer lesão de sobrecarga ser o repouso $(27,71,97)$ implica que o paciente tenha que reduzir drasticamente o número de horas de trabalho por dia ou mesmo interromper totalmente as suas actividades, o que poderá ser extremamente prejudicial em algumas profissões ou ocupações. Além disso, depois dos sintomas da doença se começarem a manifestar e dependendo da severidade dos mesmos, a intervenção cirúrgica poderá ser a única opção efectiva para 
os anular $(7,27)$, trazendo com ela os normais problemas associados a uma intervenção clínica desse tipo (a necessidade de interromper as actividades laborais, os custos relacionados com a operação, a recuperação pós-operatória, etc.). Por último, pode ainda ser acrescentado que, segundo um estudo efectuado nos Estados Unidos, 36\% dos pacientes a quem foi diagnosticado o SCC necessitam de tratamento médico durante toda a vida (113).

\section{PREVENÇÃO}

Por todos os motivos referidos anteriormente, a prevenção parece assumir uma importância capital no combate de uma doença em que os seus sintomas e os próprios tratamentos que os medeiam e controlam se apresentam como prejudiciais e limitantes para o normal desenvolvimento das tarefas profissionais e pessoais. Assim, de seguida, serão apresentadas as medidas preventivas de maior relevo para evitar o aparecimento e o desenvolvimento do SCC.

\section{Prevenção primária}

Tendo em conta que os factores de risco do SCC estão bem definidos, a prevenção primária poderá ser a estratégia de intervenção mais eficaz na tentativa de reduzir as possibilidades de desenvolvimento desta doença $(60,110)$. Deste modo, tal como na prevenção do aparecimento da grande maioria das lesões de sobrecarga, após analisar alguma da literatura existente acerca do SCC, verifica-se que as medidas preventivas centram-se, fundamentalmente, na tentativa de anular ou, pelo menos, de minimizar os efeitos nefastos dos principais factores de risco para o aparecimento e desenvolvimento desta doença de sobrecarga (para refs. ver 27, 33, 83, 86, 96, 106, 109, 123). Apesar desses factores de risco poderem ser agrupados em quatro grupos fundamentais (factores ocupacionais, pessoais, psicológicos e clínicos), as estratégias de prevenção definidas e estudadas pela literatura têm recaído, principalmente, sobre os factores ocupacionais (27).

Assim, a maioria das medidas preventivas existentes está relacionada com a correcção postural no desempenho das tarefas profissionais, procurando evitar que os indivíduos que exercem profissões consideradas de risco para o desenvolvimento do SCC utilizem posturas anti-anatómicas no cumprimento das suas tarefas laborais $(60,109)$. Desse modo, grande parte das intervenções preventivas baseia-se no encorajamento dos sujeitos a manterem o pulso em posições neutrais (61) e em reduzir os movimentos de flexãoextensão e de desvio cubital-radial da mão (123). O progresso da Ergonomia tem sido crucial nessas intervenções, possibilitando a construção de utensílios de trabalho que visam limitar a execução de movimentos anti-anatómicos e o desenvolvimento de intensidades elevadas de força (na manipulação desses utensílios) aos seus utilizadores (para refs. ver $63,82,86,96,106)$. Embora ainda exista alguma controvérsia relativamente aos efeitos das medidas ergonómicas na prevenção do SCC - que, actualmente, são cada vez mais utilizadas -, é indiscutível que a maioria dessas intervenções têm efeitos significativos no aumento do conforto no ambiente de trabalho, melhorando as condições de trabalho a que os profissionais estão sujeitos (109).

Por outro lado, o aperfeiçoamento de instrumentos e utensílios mais ergonómicos tem sido efectuado em simultâneo com o aumento de recomendações posturais, que emergem de vários estudos e investigações efectuadas com base nos movimentos e posições adoptadas por indivíduos com diferentes profissões (para refs. ver 22, 60, 62, 63, 69, 87, 117, 123). $\mathrm{O}$ maior problema que surge associado às recomendações posturais consiste na impossibilidade de as generalizar, uma vez que são específicas de cada profissão e de cada movimento. Assim sendo, apesar de, por exemplo, tanto um músico instrumentista, como um operário fabril ou um técnico administrativo (que utiliza constantemente o teclado ou o rato do computador), poderem vir a desenvolver o SCC (devido aos vários factores de risco a que estão sujeitos no cumprimento das suas tarefas profissionais), as recomendações posturais específicas que visam prevenir o aparecimento dessa doença são significativamente diferentes para cada caso (para refs. ver 28 , 62, 69). Este facto implica que seja necessário efectuar estudos particulares para cada uma das profissões/ocupações consideradas de risco para o desenvolvimento deste distúrbio musculo-esquelético. Contudo, apesar das recomendações posturais específicas variarem de movimento para movimento, há determinadas medidas genéricas de prevenção primária que podem ser aplicadas à generalidade dos 
casos. Uma dessas medidas consiste na utilização de um pulso elástico por parte dos indivíduos envolvidos em ocupações/profissões de risco para o desenvolvimento do $\operatorname{SCC}(27,84,109)$. Szabo e King (109) afirmam que o teor preventivo da utilização de pulsos elásticos se centra na manutenção do pulso numa posição neutra, uma vez que a extensão e a flexão dessa articulação - que promovem o aumento da PCC, podendo inibir a função do nervo mediano - são significativamente limitadas; ou seja, na opinião destes autores, esta medida preventiva baseiase, também ela, na limitação da utilização de posições anti-anatómicas que favorecem o aumento da PCC. Contudo, outros estudos obtiveram resultados contraditórios relativamente à utilização desta mesma medida preventiva, verificando-se que, nalgumas situações, a utilização de pulsos elásticos pode contribuir para a elevação da PCC (para refs. ver 84). Assim, apesar desta estratégia ser actualmente usada na prevenção do aparecimento do SCC (109), a literatura apresenta resultados contraditórios quanto ao seu uso, que remetem para a necessidade de ser desenvolvido um maior número de investigações visando o esclarecimento dos efeitos da utilização do pulso elástico na prevenção deste distúrbio musculo-esquelético.

Além desta medida preventiva, Lincoln et al. (61) referem que a educação acerca dos distúrbios musculo-esqueléticos relacionados com a profissão poderá desempenhar um papel importante na prevenção do SCC. Contudo, segundo estes mesmos autores, ainda não se conseguiu provar que, de forma isolada, um programa educacional tenha sido associado a qualquer tipo de efeito benéfico - o que pode ser explicado pelo facto dos estudos efectuados na tentativa de verificar o êxito dessa medida preventiva terem utilizado amostras muito reduzidas.

Para terminar a referência às estratégias de prevenção primária do SCC, é importante salientar que, segundo Szabo (108), para promover medidas preventivas racionais em trabalhadores com elevados riscos de desenvolverem esta doença musculoesquelética, tem que ser adquirida informação válida e científica acerca da associação entre a exposição a factores de risco e a neuropatia do nervo mediano. Assim, apesar dos dados confirmarem que a relação entre a profissão e o desenvolvimento do
SCC é elevada (22), ainda há um défice significativo relativamente ao conhecimento relacionado com a causalidade desta relação, sendo fundamental o desenvolvimento de estudos com o objectivo de clarificar a relação entre a ergonomia da profissão/ocupação e as lesões relacionadas com a mesma (28). Neste sentido, Barr e Barbe (7) afirmam ser importante procurar definir quais os requisitos de determinada profissão/ocupação ao nível da força e da repetição, verificando se esses requisitos ultrapassam o safe limit teórico apontado para o desenvolvimento da lesão. Apesar de terem sido efectuadas várias tentativas de averiguar e clarificar a relação exposição-resposta entre os factores de risco e o SCC, continuam a existir grandes dificuldades na medição directa das forças a que o indivíduo está sujeito, impedindo que haja um conhecimento suficiente acerca da exposição máxima aceitável perante os factores de risco (22).

Assim, a solução para que as medidas preventivas primárias, baseadas no controlo dos factores de risco ocupacionais, tenham uma maior eficácia parece residir no desenvolvimento de um número muito maior de estudos e investigações rigorosas, que permitam avaliar quantitativamente cada um desses factores de risco (60).

\section{Prevenção secundária}

O diagnóstico precoce do SCC tem sido identificado por vários autores como sendo a chave para o controlo (impedindo o agravamento a longo prazo) e tratamento da doença $(84,102)$. Segundo Mani e Gerr (68), a detecção precoce da dor tem um papel central nesse processo, sendo considerada importante para controlar os sintomas e para oferecer uma maior oportunidade de minimizar futuros riscos para os pacientes.

Assim sendo, apesar da prevenção secundária do SCC ser uma área que, até ao momento, foi pouco estudada, havendo poucos dados disponíveis para orientar essa intervenção (109), o diagnóstico precoce da doença inflacciona as possibilidades dessa prevenção ser bem sucedida.

Apesar de haver um reduzido número de estudos que exploraram a prevenção secundária deste distúrbio musculo-esquelético (109), segundo Pantukosit et al. (82), ela pode ser efectuada através de uma 
grande variedade de intervenções. Algumas dessas intervenções fundamentam-se no tratamento das condições médicas subliminares ao aparecimento do $\operatorname{SCC}(27,82)$. Assim, encorajar o paciente a deixar de fumar, diminuir a ingestão de álcool e praticar técnicas de gestão e controlo de stress são algumas sugestões referidas por Downs (27).

Pantukosit et al. (82) defendem que, para além do tratamento das condições médicas subliminares, a análise da actividade causadora dos distúrbios é fundamental para que possam ser efectuadas adaptações nessa actividade, na tentativa de evitar o reaparecimento da lesão. Assim, segundo estes autores, torna-se fundamental que a recuperação da doença seja acompanhada de um programa educativo. Nesse programa deverão ser explicadas algumas regras para evitar a reincidência do SCC, tais como a necessidade de se efectuarem pausas frequentes durante a realização da actividade causadora da lesão (o que pode permitir a recuperação em alguns tecidos de danos causados por traumas repetitivos), a explicação ao paciente acerca do impacto de uma lesão de sobrecarga e a necessidade de utilizar posições de trabalho mais ergonómicas e ferramentas mais leves (82, 109) e aderentes (63). Contudo, Lincoln et al. (61) referem que estas medidas educacionais não revelam qualquer tipo de efeito positivo quando aplicadas isoladamente.

Assim, pode-se verificar que grande parte das medidas de prevenção secundária se baseia na detecção e correcção dos factores que contribuíram para o aparecimento da doença. Desse modo, seja através de medidas educativas ou do tratamento das condições médicas subliminares, estas intervenções têm o objectivo de evitar o reaparecimento do SCC após a sua cura. Porém, é notória a falta de estudos relacionados com a prevenção secundária do SCC - onde sejam avaliados os efeitos das medidas que integram esse tipo de prevenção - (109), o que se traduz num reduzido número de dados acerca da mesma e na prevalência de opiniões contraditórias (para refs. ver 27, 61, 82).

\section{ACTIVIDADE FÍSICA E SÍNDROME DO CANAL CÁRPICO}

Apesar de alguns autores considerarem que a actividade física (AF) pode ter um papel importante na prevenção do SCC $(27,62,82,87,88)$, há poucos estudos disponíveis que permitam averiguar a sua influência na prevenção primária e secundária desta doença musculo-esquelética (109).

Relativamente à prevenção primária, alguns autores referem que a manutenção de uma boa forma aeróbia tem um papel preventivo fundamental para evitar o aparecimento de problemas musculo-esqueléticos como o SCC $(27,62,87)$. A esta opinião, Scheuerle et al. (87) acrescentam que a realização de uma mobilização articular específica, antes do desenvolvimento da tarefa manual em questão, pode evitar o aparecimento dos sintomas dolorosos característicos do SCC. Contudo, em nenhum dos casos referidos as intervenções apresentadas foram acompanhadas por resultados de estudos que comprovassem a eficácia das mesmas (para refs. ver 27, $62,87)$. Por outro lado, não são apresentadas prescrições claras acerca da forma como essas medidas devem ser implementadas; ou seja, a AF é apenas referida de forma generalizada, não sendo propostas estratégias concretas para estabelecer a sua prática (para refs. ver 27, 62, 87, 109). Assim, o papel da AF na prevenção primária do SCC ainda não foi bem estabelecido.

Quanto à prevenção secundária, Downs (27) refere que a participação em exercícios aeróbios pode ajudar a reduzir os sintomas incapacitantes originados pela doença. Também Szabo e King (109) afirmam que, depois de detectada uma lesão musculo-esquelética, é frequentemente prescrito pelo médico um programa de reabilitação que inclui exercícios de flexibilidade e de musculação. Porém, um estudo desenvolvido por Williams et al. (120) parece contrariar a opinião destes últimos autores. Nesse trabalho, que tinha como objectivo testar o impacto de um programa de exercícios de força e de flexibilidade, na tentativa de reduzir os sintomas de trabalhadores afectados pelo SCC, os resultados obtidos não permitiram comprovar os efeitos benéficos da aplicação desse programa. Este facto, inevitavelmente, levanta dúvidas acerca da viabilidade da utilização da AF como forma de prevenção secundária deste distúrbio musculo-esquelético (apesar da $\mathrm{AF}$ abranger um conjunto de medidas muito mais vasto que não se restringe a exercícios de força e de flexibilidade). Assim, tanto na prevenção primária como na prevenção secundária, verifica-se uma importante falta de estudos acerca dos efeitos da AF no SCC (109), o 
que impossibilita uma compreensão mais profunda acerca desta temática. Além disso, numa meta-análise acerca das principais intervenções preventivas para evitar o SCC, Lincoln et al. (61) verificaram que grande parte dos estudos que se debruçaram sobre os efeitos preventivos da AF no aparecimento desta doença foram baseados em amostras muito reduzidas, o que pode ter influenciado os resultados dos mesmos. As consequências conjugadas destes dois factores permitem justificar a existência de opiniões divergentes na comunidade científica acerca do papel da AF neste processo. Deste modo, tendo em conta que a literatura especializada sobre o SCC apresenta dados insuficientes e contraditórios acerca deste assunto, para procurar saber se a AF poderá ser uma medida utilizada na prevenção do SCC é necessário recorrer a literatura científica de diferentes áreas. Apesar de ser inquestionável que a prática regular de AF é benéfica para a saúde em termos gerais (5), no âmbito deste trabalho interessa verificar se ela tem potencialidades para prevenir o aparecimento do SCC. Se, tal como já foi referido, grande parte das intervenções preventivas que visam evitar o aparecimento desta doença se concentram na redução ou na anulação dos factores de risco da mesma, é importante procurar conhecer a relação entre a $\mathrm{AF}$ e esses mesmos factores. Depois de conhecer a influência que a AF pode ter nos factores de risco do SCC, será mais fácil procurar compreender se ela poderá ser utilizada como medida de intervenção preventiva para evitar essa lesão de sobrecarga. Para isso, será analisada a influência da AF nos quatro grupos de factores de risco directamente implicados no SCC. Contudo, é importante referir que apenas serão apresentados argumentos que visam demonstrar a influência preventiva da AF, não sendo apresentadas prescrições da mesma.

\section{Actividade física e factores de risco ocupacionais do SCC} São conhecidos os efeitos positivos do exercício físico, de características aeróbias, na melhoria dos índices de resistência muscular e no aumento da tolerância à fadiga (30). O treino aeróbio induz um conjunto de adaptações que aumentam a capacidade de produzir energia pelas fontes aeróbias $(30,122)$. Essas adaptações positivas ao treino aeróbio podem verificar-se quer nos praticantes de desportos aeróbios, quer nos indivíduos sedentários, ao fim de algumas semanas de treino $(5,30)$. Assim, conhecendo a relação entre a actividade física e a aptidão física (para refs. ver 13, 67), facilmente se compreende que os indivíduos com fracos índices de aptidão física tenham tendência em fatigar-se mais precocemente. Segundo Branco e Pereira (17), essa tendência é mais acentuada na execução de tarefas repetitivas, o que na consecução de actividades laborais leva frequentemente à adopção de posturas incorrectas. Tendo em consideração que a utilização de posições anti-anatómicas é um dos factores de risco determinantes para o aparecimento e desenvolvimento do SCC $(28,82,91)$ e que, por isso, algumas das medidas preventivas primárias mais implementadas consistem na tentativa de evitar a utilização de posturas anti-anatómicas (para refs. ver 60, 109), a AF pode constituir-se como uma estratégia de prevenção primária dessa doença. Essa capacidade de promover uma intervenção preventiva pode ser justificada pelo facto do desenvolvimento dos níveis de aptidão física poder ser efectuado através da prática regular de AF, desencorajando a utilização de posturas anti-anatómicas no trabalho. Assim, a AF pode desempenhar um papel importante na melhoria da resistência geral ao trabalho muscular, reduzindo o aparecimento da fadiga (para refs. ver 30,122), o que, simultaneamente, parece reduzir as possibilidades de se utilizarem posturas anti-anatómicas na realização das tarefas laborais (17).

A repetição e a intensidade da força subjacentes à realização das tarefas laborais constituem-se como outros dois factores de risco ocupacionais preponderantes para o desenvolvimento do SCC $(28,66,82)$. Se, por um lado, os efeitos desses factores dependem do facto das suas intensidades ultrapassarem (ou não) o safe limit teórico apontado para o desenvolvimento da lesão (7), por outro lado, a AF pode contribuir para o aumento da capacidade do organismo tolerar esforços e estender os seus limites fisiológi$\cos (30,72)$. Assim, é coerente especular que a $\mathrm{AF}$ pode permitir uma maior tolerância a esses factores de risco, ampliando os valores máximos da exposição aceitável - safe limit - de cada indivíduo. Considerando que o SCC atinge indivíduos que, normalmente, têm ocupações em que é necessária a realização de um elevado número de tarefas manuais onde a articulação do pulso é fortemente solicitada (32, 44, 87, 123), é importante referir alguns efeitos 
particulares da exercitação específica dos MS. Tendo em conta que uma mesma carga desenvolvida pelos MS origina uma maior subida de pressão arterial $(\mathrm{PA})$ e da frequência cardíaca (FQ) do que se efectuada pelos membros inferiores (29), a utilização exclusiva dos MS na consecução das tarefas laborais (particularmente nas tarefas em que é necessária a aplicação de elevadas intensidades de força) origina maiores exigências cardíacas e maior subida tensional do que se a mesma carga fosse repartida por um maior número de segmentos corporais. Desse modo, segundo Mitchell e Raven (72), indivíduos dependentes da utilização exaustiva dos MS devem insistir no treino (com características aeróbias) desses segmentos corporais, no sentido de serem promovidas adaptações crónicas na FQ e PA, que lhes permitam uma maior tolerância à fadiga causada por essa solicitação localizada e específica - advindo daí as vantagens anteriormente referidas.

Desse modo, se a AF geral aparenta exercer um poder preventivo no SCC, a exercitação específica dos MS parece, pela sua maior especialização face às tarefas ocupacionais em questão, potenciar essa capacidade preventiva.

Assim, relativamente aos factores de risco ocupacionais, existem motivos para se encarar a AF como uma possível base para a prevenção primária do SCC: a sua relação com a postura adoptada nas actividades laborais e a possibilidade de aumentar a tolerância do organismo aos efeitos da repetitividade do movimento e da intensidade da força subjacente a determinada tarefa profissional, justificam essa possibilidade. Por outro lado, tal como é sugerido no "mundo do desporto" (para refs. ver 46, 71, 85), o desenvolvimento de uma exercitação mais específica e direccionada para a tarefa ocupacional em questão parece potenciar esses efeitos preventivos, fortalecendo, de forma mais eficaz, as estruturas especificamente solicitadas e aumentando - ainda mais - a resistência ao aparecimento de possíveis doenças musculo-esqueléticas (tais como o SCC), face à exposição de factores de risco. A tudo isto pode ser acrescentado o facto da AF poder promover um aumento da condição física (30) que, por sua vez, diminui a predisposição para a ocorrência de lesões musculo-esqueléticas em geral (47) - tipo de lesões onde se pode incluir o SCC.
Actividade física e factores de risco biológicos do SCC Um elevado IMC (IMC>30) parece ser um factor de risco determinante para o aparecimento e desenvolvimento do SCC $(11,54,66,78,99,105,111,119)$. Este facto pode ser justificado tanto pela relação directa que apresenta com o aumento da PCC (8), como pelas consequências psíquicas que a obesidade frequentemente acarreta (para refs. ver 4) e que, muitas vezes, se enquadram nos factores de risco psicológicos para o desenvolvimento da doença. Tendo em conta que a maioria dos estudos epidemiológicos demonstram que o aumento de peso ao longo da vida está mais relacionado com a diminuição dos hábitos de exercício, do que com o aumento da ingestão calórica (5), facilmente se compreende que a AF pode ser utilizada na prevenção e terapêutica da obesidade ligeira ou moderada (4). Ao promover o dispêndio energético durante a sua realização (5), a AF tem um contributo importante na redução ou manutenção do IMC no indivíduo. De facto, toda a AF comporta um custo calórico, sendo possível nas actividades desportivas estereotipadas (como, por exemplo, a marcha, a natação, o remo, a corrida, etc.) prever com rigor esse consumo calórico por métodos indirectos (para refs. ver 4, 122). Assim, a AF poderá estar directamente relacionada com a perda de peso e, desse modo, representa uma medida preventiva ao aparecimento de um IMC elevado, contribuindo para a minimização e controlo de um dos factores de risco biológicos mais importantes para o aparecimento do SCC.

Para além disso, é importante salientar que a AF tem também efeitos significativos na potenciação dos resultados positivos das dietas alimentares, contribuindo para a manutenção do bem-estar psíquico do paciente (que é, muitas vezes, posto em causa) durante a realização da dieta e para uma maior adesão ao programa alimentar sugerido. Desse modo, para além da própria AF poder desempenhar um papel directo na redução do peso corporal, tem também efeitos benéficos, indirectos, na influência das dietas alimentares, tornando-as mais eficazes (79). É também importante salientar que a realização regular de AF poderá estar relacionada com a diminuição de peso corporal através da perda preferencial de massa gorda (4) e não, tal como acontece sempre que o organismo entra em balanço energético negati- 
vo (tal como, por exemplo, no seguimento de uma dieta alimentar com a finalidade de perder peso corporal), associado à perda conjunta de massa magra e de massa gorda (5); ou seja, a AF regular permite uma redução do IMC à custa, fundamentalmente, da redução da massa gorda. Assim, apesar das variações de peso à custa, unicamente, da massa gorda serem aparentemente impossíveis (34), a acção trófica da AF sobre os músculos exercitados contrapõe-se à perda de massa muscular causada pelo balanço energético negativo necessário para que haja uma diminuição da massa corporal (5). Assim, a AF poderá permitir solucionar este factor de risco para o SCC o elevado IMC - sem penalizar os valores máximos da exposição aceitável do indivíduo a factores de risco, tais como a força e a repetição - uma vez que a perda de massa magra está normalmente associada a uma maior fragilidade da condição física e, por isso, à diminuição da capacidade de adaptação aos estímulos diários e a um aparecimento mais precoce da fadiga (para refs. ver 14).

Por último, é pertinente referir que, quando o principal objectivo da realização de AF é a perda de peso, devem-se escolher exercícios que originam consumos de oxigénio mais elevados - exercícios de longa duração, elevada intensidade e que solicitem a maior quantidade de massa muscular possível (18). Porém, tendo em conta que a maioria dos indivíduos com IMC elevado apresentam uma condição física reduzida, a intensidade da sua exercitação física deverá ser reduzida, impedindo também o abandono por desmotivação $(4,18)$. Assim, os exercícios a escolher deverão ser prolongados, pouco exigentes no que respeita à intensidade, e mobilizar amplas massas musculares - por exemplo, a marcha, a corrida, o ciclismo, a dança, etc. $(4,18,122)$.

\section{Actividade física e factores de risco psicológicos do SCC}

Os efeitos benéficos da AF não devem ser vistos exclusivamente do ponto de vista somático (57). Esses efeitos contemplam também aspectos psicológicos importantes que, por sua vez, medeiam algumas reacções somáticas (74). Os benefícios psicológicos que advêm da realização de AF parecem evidentes, sendo referidos frequentemente por vários autores (para refs. ver 10, 90, 115, 121). Apesar das relações exactas entre a AF e os benefí- cios psicológicos não estarem totalmente compreendidas, devido à elevada complexidade de tal relação (24), segundo Berger e Mclnman (10) pode-se concluir que a AF realizada de forma habitual pode estar associada à promoção de bem-estar psicológico. Esse bem-estar está relacionado, geralmente, ao grau de satisfação do indivíduo com a sua vida (24). Um estudo desenvolvido no Reino Unido (1) sugere que as pessoas que praticam exercício físico têm uma maior percepção de auto-satisfação. Contudo, essa investigação deixa em aberto a possibilidade desta relação poder ser lida em sentido contrário, ou seja, as pessoas que se percebem a si próprias como tendo boa saúde são mais atraídas para o exercício físico. Além da influência que o exercício físico parece ter no bem-estar psicológico, Cruz et al. (24) resumem alguns benefícios potenciais da AF na vida geral, sendo importante realçar aqueles que se podem relacionar, de alguma forma, com o SCC. Assim, a AF aumenta o auto-controlo e a eficiência no trabalho; por outro lado, diminui o sentimento de ira/irritação e ansiedade, a depressão e os erros no trabalho. Tal como foi descrito atrás, vários estudos apontam para o facto do reduzido bem-estar psicológico, da reduzida satisfação no trabalho, do reduzido autocontrolo, da ansiedade/pressão, do stress e de um trabalho monótono, parecerem estar associados ao maior risco de desenvolvimento de lesões musculoesqueléticas de sobrecarga $(12,44,59)$. Assim, face aos efeitos psicológicos da AF (as suas virtudes na minimização ou anulação destes factores são bem visíveis) torna-se fácil compreender as suas potencialidades na intervenção preventiva primária e secundária do SCC.

\section{Actividade física e factores de risco clínicos do SCC}

Tal como referido, a DM é o factor de risco clínico mais estudado e que parece estar mais associado ao desenvolvimento do SCC $(8,28)$. Já antes da descoberta da insulina (no início do século XX), a AF era considerada como benéfica para os doentes com esta perturbação metabólica (79). Actualmente, os benefícios da AF na prevenção e controlo da DM estão comprovados (36). Assim, Barata e Lisboa (6) resumem os efeitos positivos da AF nos diabéticos referindo que esta facilita a redução do peso, promove uma maior sensibilidade periférica aos efeitos da 
insulina, melhora o perfil lipídico e reduz a hipertensão arterial coexistente.

Apesar do papel da AF ser fundamental nos pacientes com DM, existem regras padronizadas para a sua prescrição que devem, acima de tudo, ser individualizadas em função da gravidade da doença e das complicações existentes (6). Contudo, de uma forma geral, as actividades quantificáveis e reprodutíveis (estereotipadas) parecem ser as mais aconselhadas, uma vez que permitem uma prescrição mais rigorosa das cargas (39). Por outro lado, Pérez e Cros (83) acrescentam que as actividades ideais parecem ser as que utilizam maior massa muscular, com características aeróbias, e que se podem manter por um período prolongado no tempo, tais como, por exemplo, a marcha, a natação, a dança ou o ciclismo.

Para além da AF poder contribuir para a redução de um factor de risco clínico determinante como a DM, ela também pode estimular o aumento da massa óssea, promovendo uma maior resistência mecânica do osso (15). De facto, a AF tem um papel que poderá ser decisivo na estimulação dos osteoblastos - particularmente após os 35 anos, idade média em que a actividade osteoclástica começa a ser responsável pela rarefacção do tecido ósseo (para refs. ver 16, 23). Desse modo, enquanto que a imobilização ou a ausência de carga provocam o aumento da eliminação urinária do cálcio e a redução óssea acelerada, a acção muscular subjacente à AF promove forças mecânicas sobre o osso que permitem, por estimulação osteoblástica, manter ou aumentar a sua massa (15). Naturalmente, esse aumento da massa óssea corresponde a um incremento da resistência mecânica do tecido ósseo mais estimulado, reduzindo o risco de fracturas (23). Sabendo que a fractura do pulso é apontada como um antecedente de risco para o aparecimento do SCC (53) -, é coerente supor que a prática regular de AF (desde que as cargas dessa actividade estimulem essa articulação) pode desempenhar um papel importante na redução das possibilidades de surgimento desse factor de risco.

Perante a influência positiva que a AF aparenta ter na prevenção e no tratamento de alguns dos factores de risco mais significativos para o aparecimento e desenvolvimento do SCC, parece ser lógica a sua integração nas medidas preventivas primárias e secundárias. Porém, como a acção preventiva global da AF é mais eficaz do que a sua componente curativa (5), no caso da sua intervenção específica sobre os factores de risco do SCC, a sua acção parece também mais benéfica e viável quando utilizada como medida de prevenção primária.

\section{CONCLUSÕES E SUGESTÕES PARA INVESTIGAÇÕES FUTURAS}

Perante a compreensão da etiologia e dos principais sintomas do SCC, há todo o interesse em concentrar esforços na prevenção deste distúrbio musculoesquelético. Desse modo, vários autores têm-se dedicado a investigar os factores de risco do SCC e possíveis estratégias de prevenção. Todavia, têm surgido vários problemas que obstaculizam a criação e o desenvolvimento de medidas preventivas primárias e secundárias. Um desses problemas prende-se com a quantificação das cargas físicas que promovem o aparecimento do SCC; ou seja, factores de risco determinantes como a intensidade e a duração da força exercida, a repetição do movimento subjacente à realização de uma tarefa e a postura anti-anatómica utilizada ainda não foram rigorosamente quantificados. Não se conseguiu delimitar (de forma clara e rigorosa) o safe limit a partir do qual esses factores passam a sujeitar os tecidos a cargas que ultrapassam os seus limites fisiológicos, constituindo-se como uma potencial causa para o desenvolvimento do SCC.

Além desta dificuldade, outra adversidade que tem retardado os progressos na prevenção do SCC está relacionada com os vários métodos utilizados para definir os factores de risco desta doença. Essa multiplicidade metodológica tem contribuído para a proliferação de algumas contradições relativamente a esses factores e, simultaneamente, para alguma confusão na classificação e interpretação dos mesmos. Obviamente que estes problemas têm constrangido e limitado o estudo e a compreensão da relação exposição-resposta, ou seja, da relação factores de riscoSCC. O estabelecimento incompleto dessa relação reflecte-se ao nível do desenvolvimento das estratégias preventivas da doença. Apesar disso, os factores de risco aparentemente mais significativos estão bem definidos pela literatura. Contudo, é importante referir que essa definição acompanha a tendência da literatura em se concentrar, preferencialmente, sobre os factores de risco ocupacionais, deixando frequen- 
temente os restantes factores para segundo plano. O conhecimento dos principais factores de risco tem facilitado o desenvolvimento de algumas estratégias de prevenção, que se baseiam eminentemente na minimização e na tentativa de anulação desses mesmos factores. Apesar da AF parecer ter um papel importante na prevenção de grande parte dos factores de risco determinantes, tendo sido apresentados exemplos da sua possível influência positiva, a literatura especializada não a tem encarado como uma medida preventiva potencialmente eficaz. Se, por um lado, os estudos que visam analisar a AF como medida preventiva são extremamente reduzidos, por outro lado, a sua grande maioria apresenta amostras muito limitadas, não permitindo obter dados rigorosos acerca da influência da mesma na prevenção do SCC. Assim, apesar de vários autores referirem a AF como medida preventiva primária, verificam-se algumas divergências nas estratégias referidas para a sua implementação - reflexo da falta de investigações desenvolvidas relativamente a esse assunto.

A AF parece revestir-se de um elevado potencial preventivo (primário e secundário), permitindo controlar e minimizar (ou mesmo anular) alguns dos principais factores de risco - por exemplo, a DM, um elevado IMC, os efeitos nefastos da utilização de posturas anti-anatómicas, de movimentos repetitivos e o reduzido bem-estar psicológico.

Para finalizar é importante referir que, apesar da AF ainda não ter motivado a realização de um número suficiente de estudos que permitam conhecer as suas potencialidades como uma medida preventiva determinante do SCC, parecem existir justificações para que sejam desenvolvidas investigações mais rigorosas acerca dos seus efeitos na prevenção primária e secundária do SCC. Porém, mesmo com um insuficiente número de estudos realizados, é possível inferir que, face às suas comprovadas capacidades de prevenir e de tratar alguns dos factores de risco mais directamente associados com o SCC, a AF, mesmo que de forma indirecta, pode reduzir o risco de aparecimento e de desenvolvimento do SCC.

\author{
CORRESPONDÊNCIA \\ José Alberto Duarte \\ Laboratório de Bioquímica \\ e Morfologia Experimental \\ Faculdade de Ciências do Desporto \\ e de Educação Física \\ Universidade do Porto \\ Rua Dr. Plácido Costa, 91 \\ 4200 - 450 Porto, Portugal \\ jarduarte@fcdef.up.pt
}




\section{REFERÊNCIAS BIBLIOGRÁFICAS}

1. Allied Dumbar National Fitness Survey (1992). Main Findings. London: Sport Council and Health Education Authority.

2. Armstrong TJ, Buckle P, Fine LJ, Hagberg M, Jonsson B, Kilbom A, Kuorinka IAA, Silverstein BA, Sjogaard G, Viikari-Juntura ERA (1993). A conceptual model for workrelated neck and upper-limb musculoskeletal disorders. Scand J Work Environ Health. 19, 73-84

3. Armstrong TJ, Chaffin DB (1979). Carpal tunnel syndrome and selected personal attributes. J Occup Med. 21, 481-486.

4. Atkinson RL, Walberg-Rankin J (1993). Physical activity, fitness and severe obesity. In C Bouchard, R Shephard, T Stephens (Eds). Physical Activity, Fitness, and HealthConsensus Statement. Champaign: Human Kinetics, 69.

5. Barata $\mathrm{T}(1997)$. Benefícios da actividade física regular. In $\mathrm{T}$ Barata (Ed). Actividade Física e Medicina Moderna. Odivelas: Europress, 132-152.

6. Barata T, Lisboa PE (1997). Diabetes e actividade física. In T Barata (Ed). Actividade Física e Medicina Moderna. Odivelas: Europress, 287-297.

7. Barr AE, Barbe MF (2002). Pathophysiological tissue changes associated with repetitive movement: a review of the evidence. Phys Ther. 82, 173-187.

8. Becker J, Nora DB, Gomes I, Stringari FF, Seitensus R, Panosso JS, Ehlers JAC (2002). An evaluation of gender, obesity, age and diabetes mellitus as risk factors for carpal tunnel syndrome. Clin Neurophysiol. 113, 1429-1434.

9. Bekkelund S, Pierre-Jerome C, Torbergsen T, Ingebrigtsen $\mathrm{T}$ (2001). Impact of occupational variables in carpal tunnel syndrome. Acta Neurol Scand. 103, 193-197.

10. Berger BG, Mclnman A (1993). Exercise and the quality of life. In RN Singer, M Murphey, LK Tennant (Eds) Handbook of Research on Sport Psychology. New York: Macmillan, 729760.

11. Billi A, Catalucci A, Barile A, Masciocchi C (1998). Joint impingement syndrome: clinical features. Eur J Radiol. 27, 39-41.

12. Bongers PM, de Winter CR, Kompier MA, Hildbrandt VH (1993). Psychosocial factors at work and musculoskeletal disease. Scand J Work Environ Health. 19, 297-312.

13. Borms J (1991). Exercício físico, aptidão física e o novo paradigma de saúde. In J Bento, A Marques (Eds). Actas das Jornadas Científicas: Desporto. Saúde. Bem-Estar. Porto: FCDEF-UP, 111-118.

14. Bouchard C, Shephard R (1993): Physical activity, fitness, and health: the model and key concepts. In C Bouchard, R Shephard, T Stephens (Eds). Physical Activity, Fitness, and Health - Consensus Statement. Champaign: Human Kinetics, $11-24$.

15. Branco J (1997). Massa óssea e actividade física. In T Barata (Ed). Actividade Física e Medicina Moderna. Odivelas: Europress, 298-303.

16. Branco JC, Matos ACA, Silva JC (1998): Metabolismo fosfo-cálcio. Geriatria. 1, 30-39.

17. Branco J, Pereira G (1997). Lombalgias e actividade física. In T Barata (Ed). Actividade Física e Medicina Moderna. Odivelas: Europress, 304-309.

18. Bray GA (1990). Exercise and obesity. In Bouchard C, Shephard R, Stephens T, Sutton JR, McPherson BD (Eds). Exercise, Fitness and Health $-a$ Consensus of Current
Knowledge. Champaign: Human Kinetics, 499-510.

19. Brouwer B, Mazzoni C, Pearce W (2001). Tracking ability in subjects symptomatic of cumulative trauma disorder: does it relate to disability? Ergonomics 44, 443-456.

20. Buckle PW (1997). Work factors and upper limb disorders. BMJ. 315, 1360-1363.

21. Cakir M, Samancit N, Balcit N, Balci MK (2003). Musculoskeletal manifestations in patients of thyroid disease. Clin Endocrinology 59, 162-167.

22. Ciriello VM, Snook SH, Webster BS, Dempsey P (2001) Psychophysical study of six hand movements. Ergonomics 44, 922-936.

23. Constantini NW, Warren MP (1994). Special problems of the female athlete. Clin Rheumathol. 8, 199-219.

24. Cruz J, Machado P, Mota M (1996). Efeito e benefícios psicológicos do exercício e da actividade física. In Cruz J (Ed). Manual de Psicologia do Desporto. Braga: Sistemas Humanos e Organizacionais, 91-116.

25. Cullum DE, Molloy CJ (1994). Occupation and the carpal tunnel syndrome. Med J Aust. 161, 552-554.

26. De Krom MC, Knipschild PG, Kester AD, Thijs CT, Boekkooi PF, Spaans F (1990). Risk factors for carpal tunnel syndrome. J Clin Epidem. 132, 1102-1110.

27. Downs DG (1997). Nonspecific work-related upper extremity disorders. Am Fam Physician. 55, 1296-1302.

28. Fagarasanu M, Kumar S (2002). Carpal tunnel syndrome due to keyboarding and mouse tasks: a review. Int J Industr Erg. (In press).

29. Fagard RH, Tipton CM (1993). Physical activity, fitness and hypertension. In Bouchard C, Shephard R, Stephens T (Eds). Physical Activity, Fitness, and Health - Consensus Statement. Champaign: Human Kinetics, 41.

30. Faulkner JA, Green HJ, White TP (1993). Physical activity and skeletal muscle. In Bouchard C, Shephard R, Stephens $\mathrm{T}$ (Eds). Physical Activity, Fitness, and Health-Consensus Statement. Champaign: Human Kinetics, 55-56.

31. Feuerstein M, Armstrong T, Hickey P, Lincoln A (1997). Computer keyboard force and upper extremity symptoms. J Occup Environ Med. 39, 1144-1153.

32. Feuerstein M, Miller VL, Burrel ML, Berger L (1998). Occupational upper extremity disorders in the federal workforce: prevalence, health care expenditures, and patterns of work disability. J Occup Environ Med. 40, 546-555.

33. Forsman M, Hansson GA, Medbo L, Asterland P, Engstrom $\mathrm{T}$ (2002). A method for evaluation of manual work using synchronised video recordings and physiological measurements. Appl Ergonomics 33, 533-540.

34. Garrow JS, Webster J (1985): Quetelet's index $\left(\mathrm{W} / \mathrm{H}^{2}\right)$ as a measure of fatness. Int J Obesity 9, 147-153.

35. Gerritsen A, Uitdehaag B, Geldere D, Scholten R, Vet H, Bouter L (2001). Systematic review of randomized clinical trials of surgical treatment for carpal tunnel syndrome. BJS. 88, 1285-1295.

36. Giacca A (1993). Physical activity, fitness and type 1 diabetes. In Bouchard C, Shephard R, Stephens T (Eds). Physical Activity, Fitness, and Health - Consensus Statement. Champaign: Human Kinetics, 65-66.

37. Goldstein B, Goetz L, Young J (1998). Treatment of repetitive motion disorders. In Shankar K (Ed). Exercise Prescription. Philadelphia: Haley \& Belfus, 363-401.

38. Gottlieb S (2001). US employer agrees to stop genetic testing. BMJ. 322, 449. 
39. Gudat U, Berger M, Lefèvre PJ (1993). Physical activity, fitness and non-insulin-dependent (type II) diabetes in physical activity. In Bouchard C, Shephard R, Stephens T (Eds). Physical Activity, Fitness, and Health - Consensus Statement. Champaign: Human Kinetics, 66-67.

40. Hadler M (1997). Carpal tunnel syndrome, diagnostic conundrum. J Rheumatol. 24, 417-419.

41. Hagberg M (1997). ABC of work-related disorders. Neck and harm disorders. BMJ. 313, 419-422.

42. Hales TR, Bernard BP (1996). Epidemiology of work-related musculoskeletal disorders. Orthop Clin North Am. 27, 679-709.

43. Hélbert L, Camirand D (1996). Anatomie appliquée du poignet et de la main. In Le Corps et ses Mouvements. Canada: Édition Saint-Martin, 77-115.

44. Himmelstein JS, Feuerstein M, Stanek EJ (1995). Workrelated upper-extremity disorders and work disability: clinical and psychosocial presentation. J Occup Environ Med. 37, 1278-1286.

45. Hirasawa Y, Ogura T (2000). Carpal tunnel syndrome in patients on long term haemodialysis. Scand J Plast Reconstr Hand Surg. 34, 373-381.

46. Horta L (1995). Prevenção de Lesões no Desporto. In Horta L (Ed). Lisboa: Editorial Caminho, 281-378.

47. Horta L (1997). As lesões musculoesqueléticas. In Barata $\mathrm{T}$ (Ed). Actividade Física e Medicina Moderna. Odivelas: Europress, 324-345.

48. Jeng O, Radwin NG, Rodriguez AA (1994). Functional psychomotor deficits associated with carpal tunnel syndrome. Ergonomics 37, 1065-1069.

49. Keir PJ, Bach JM, Rempel DM (1998). Effects of finger posture on carpal tunnel pressure during wrist motion. $J$ Hand Surg. 23, 1004-1009.

50. Keir PJ, Wells RP (1999). Changes in geometry of the finger flexor tendons in the carpal tunnel with wrist posture and tendon load: an MRI study on normal wrists. Clin Biom. 14, 635-645.

51. Keyserling MW, Stetson DS, Silverstein BA, Brouwer ML (1993). A checklist for evaluating ergonomic risk factors associated with upper extremity cumulative trauma disorders. Ergonomics 36, 807-831.

52. Klein I, Ojamaa K (2000). Thyroid (neuro)myopathy. Lancet 356, 654.

53. Kouyoumdjian JA, Morita MPA, Rocha PRF, Miranda RC, Gouveia GM (2000). Body mass index and the carpal tunnel syndrome. Arch Neuro. 58, 252-256.

54. Kouyoumdjian JA, Zanetta DM, Morita MPA (2002). Evaluation of age, body mass index, and wrist index as risk factors for carpal tunnel syndrome severity. Muscle Nerve 25, 93-97.

55. Kumar R, Gaerlan MS, Burgess T (2000). Carpal tunnel syndrome in the presence of wrist flexion and absence of repetitive movements. J Back Musc Rehabil. 14, 127-131.

56. Lam N, Thurston A (1998). Association of obesity, gender, age and occupation with carpal tunnel syndrome. Aust $N \mathrm{Z}$ J Surg. 68, 190-193.

57. Landers DM, Petruzzello SJ (1993). Physical activity, fitness and anxiety. In Bouchard C, Shephard R, Stephens T (Ed). Physical Activity, Fitness and Health - Consensus Statement. Champaign: Human Kinetics, 80-81.

58. Lange H (1999). Carpal tunnel syndrome caused by the palmaris profundus muscle. Scand J Plast Reconstr Hand
Surg. 33, 251-252.

59. Leclerc A, Franchi P, Cristofari MF, Delemotte B, TeysierCotte C, Touranchet A (1998). Carpal tunnel syndrome and work organisation in repetitive work: a cross sectional study in France. J Occup Environ Med. 55, 180-187.

60. Lin ML, Radwin RG (1997). A single metric for quantifying biomechanical stress in repetitive motions and exertions. Ergonomics 40, 543-558.

61. Lincoln AE, Vernick JS, Ogaitis S, Smith GS, Mitchell CS, Agnew J (2000). Interventions for the primary prevention of work-related carpal tunnel syndrome. Am J Prev Med. 18, 37-50.

62. Liu S, Hayden GF (2002). Maladies in musicians. South Med J. 95, 727-734.

63. Lowe B, Freivalds A (1999). Effect of carpal tunnel syndrome on grip force coordination on hand tools. Ergonomics 42, 550-564.

64. Luttgens K, Hamilton N (1997). The upper extremity: elbow, forearm, wrist, and hand. In Kinesiology - Scientific Basis of Human Motion. Dubuque: Brown \& Benchmark Publishers, 150-172.

65. Macfarlane GJ, Hunt IM, Silman AJ (2001). Role of mechanical and psychosocial factors in the onset of forearm pain: prospective population based study. BMJ 321, 676-679.

66. Macfarlane GJ (2001). Identification and prevention of work-related carpal tunnel syndrome. Lancet 357, 1146.

67. Maia J, Lopes V, Morais F (2001). Actividade física e aptidão física associada à saúde: um estudo de epidemiologia genética em gémeos e suas famílias realizado no Arquipélago dos Açores. Porto: FCDEF-UP, Direcção Geral de Educação Física e Desporto da Região Autónoma dos Açores, 21-40.

68. Mani L, Gerr F (2000). Work-related upper extremity musculoskeletal disorders. J Occup Environ Med. 27, 845-864.

69. Marklin RW, Monroe JF (1998). Quantitative analysis of wrist motion in bone-trimming jobs in the meat packing industry. Ergonomics 41, 227-237.

70. Marshall MM, Mozrall R, Shealy JE (1999). The effects of complex wrist and forearm posture on wrist range of motion. Human Factors 41, 205-213.

71. Massada, L (2000): Lesões Típicas do Desportista. Lisboa: Editorial Caminho, 37-122.

72. Mitchell JH, Raven PB (1993). Cardiovascular adaptation to physical activity. In Bouchard C, Shephard R, Stephens T (Eds). Physical Activity, Fitness, and Health - Consensus Statement. Champaign: Human Kinetics, 41-42.

73. Moore JS, Garg A (1994). Upper extremity disorders in a pork processing plant: relationships between job risk factors and morbidity. Am Ind Hyg Assoc J. 55, 703-715.

74. Morgan WP (1993). Physical activity, fitness and depression. In Bouchard C, Shephard R, Stephens T (Eds). Physical Activity, Fitness, and Health - Consensus Statement. Champaign: Human Kinetics, 80.

75. Nathan PA, Keniston RC, Myers LD, Meadows KD (1992). Longitudinal study of median nerve sensory conduction in industry relationship to age, gender, hand dominance, occupational hand use, and clinical diagnosis. J Hand Surg. 17, 850-857.

76. Nathan PA, Keniston RC, Myers LD, Meadows KD, Lockwood RS (1999). Natural history of median nerve conduction in industry: relationship to symptoms and carpal tunnel syndrome in 558 hands over 11 years. Muscle Nerve 21, 711-721. 
77. Nelson JE, Treaster DE, Marras WS (2000). Finger motion, wrist motion and tendon travel as a function of keyboard angles. Clin Biom. 15, 489-498.

78. Nordstrom DL, Vierkant RA, DeStefano F, Layde PM (1997). Risk factors for carpal tunnel syndrome in a general population. J Occup Environ Med. 54, 734-740.

79. Nunes, L (1999). A Prescrição da Actividade Física. Lisboa: Editorial Caminho, 31-46, 183-206.

80. Ozkul Y, Sabuncu T, Yazgan P, Nazligul Y (2001). Local insulin injection improves median nerve regeneration in NIDDM patients with carpal tunnel syndrome. Eur J Neurol. 8, 329-334.

81. Palumbo CF, Szabo RM, Olmsted SL (2000). The effects of hypothyroidism and thyroid replacement on the development of carpal tunnel syndrome. J Hand Surg. 25, 734-739.

82. Pantukosit S, Petchkrua W, Stiens S (2001). Intersection syndrome in Buriram hospital - a 4-yr prospective study. Am J Phys Med Rehabil. 80, 656-661.

83. Pérez AP, Cros MR (1996). Ejercicio físico en diversos transtornos metabólicos: diabetes, dislipemia y obesidad. In Grima JR (Ed). Prescription de Ejercicio Físico para la Salud. Barcelona: Editorial Paidotribo.

84. Rempel DM, Harrison RJ, Barnhart S (1992). Work-related cumulative trauma disorders of the upper extremity. JAMA 267, 838-842.

85. Ribeiro B (1992). O Treino do Músculo. In Horta L (Ed). Lisboa: Editorial Caminho, 79-83.

86. Ro J, Jacobs K (1997). Wrist postures in video display terminal operators (VDT) using different keyboards. Work 9, 255-266.

87. Scheuerle J, Guilford AM, Habal MB (2000). Work-related cumulative trauma disorders and interpreters for the deaf. Appl Occup Environ Hyg. 15, 429-434.

88. Sequeira W (1999). Yoga in treatment of carpal tunnel syndrome. Lancet 353, 689-690.

89. Seradge H, Jia YC, Owens W (1995). In vivo measurement of carpal tunnel pressure in the functioning hand. $J$ Hand Surg. 20, 855-859.

90. Seraganian P (1993). Current status and future directions in the field of exercise psychology. In Seraganian P (Ed). Exercise Psychology - the influence of physical exercise on psychological processes. New York: John Wiley, 39-121.

91. Serina E, Tal R, Rempel D (1999). Wrist and forearm postures and motions during typing. Ergonomics 42, 938-951.

92. Sharma SD, Smith EM, Hazleman BL, Jenner JR (1997). Thermographic changes in keyboard operators with chronic forearm pain. BMJ 314, 118.

93. Silverstein BA, Fine LJ (1991). Cumulative trauma disorders of the upper extremity. J Occup Med. 33, 642-644.

94. Silverstein BA, Fine LJ, Armstrong TJ (1987). Occupational factors and carpal tunnel syndrome. Am J Industr Med. 11, 343-358.

95. Silverstein BA, Welp E, Nelson N, Kalat J (1995). Claims incidence of work related disorders of the upper extremities: Washington State, 1987 trough 1995. Am J Pub Health 88, 1827-1833.

96. Smith MJ, Karsh BT, Conway FT (1998). Effects of a split keyboard design and wrist rest on performance, posture and comfort. Human Factors 40, 324-336.

97. Sperryn P (1994). Overuse injury in sport. BMJ 308, 14301432.

98. Stabler A, Heuck A, Reiser M (1997). Imaging of the hand: degeneration, impingement and overuse. Eur J Radiol. 25, 118-128.

99. Stallings SP, Kasdan ML, Soergel TM, Corwin HM (1997). A case-control study of obesity as a risk factor for carpal tunnel syndrome in a population of 600 patients presenting for independent medical examination. J Hand Surg. 22, 211-215.

100. Stevens JC, Smith BE, Weaver AL, Bosch EP, Deen HG, Wilkens JA (1999). Symptoms of 100 patients with electromyographically verified carpal tunnel syndrome. Muscle Nerve 22, 1448-1456.

101. Stevens JC, Witt JC, Smith BE, Weaver AL (2001). The frequency of carpal tunnel syndrome in computer users at a medical facility. Neuro. 56, 1568-1570.

102. Stiens SA, Haselkorn JK, Peters JD (1996). Rehabilitation intervention for patients with upper extremity dysfunction: challenges of outcome evaluation. Am J Industr Med. 29, 590-601.

103. Stumpp D, Sparks P (1997). Screening and medical surveillance for cumulative trauma disorders. In Erdil M, Dickerson OB (Eds). Cumulative Trauma Disorders: Prevention, Evaluation, and Treatment. New York: Van Nostrand Reinhold.

104. Sugimoto H, Mijaji O, Oshaea T (1994). Carpal tunnel syndrome: evaluation of median nerve circulation with dynamic contrast-enhanced MR imaging. Radiology 190, 459-466.

105. Sungpet A, Suphachatwong C, Kawinwonggowit V (1999). The relationship between body mass index and the number of sides of carpal tunnel syndrome. J Med Assoc Thai. $82,182-185$.

106. Swanson NG, Galinsky TL, Cole LL, Pan CS, Sauter SL (1997). The impact of keyboard design on comfort and productivity in a text entry task. Appl Ergonomics 28, 9-16.

107. Szabo RM (1989). Stress carpal tunnel pressures in patients with carpal tunnel syndrome and normal patients. J Hand Surg. 14, 624-627.

108. Szabo RM (1998). Carpal tunnel syndrome as a repetitive motion disorder. Clin Orthop Research 351, 78-89.

109. Szabo RM, King KJ (2000). Repetitive stress injury: diagnosis or self-fulfilling prophecy? J Bone Joint Surg. 82, 1314-1322.

110. Tanaka S, McGlothlin JD (1993). A conceptual model for prevention of work-related carpal tunnel syndrome (CTS). Int J Industr Ergon. 11, 87-107.

111. Tanaka S, Wild DK, Cameron LL, Freund E (1997). Association of occupational and nonoccupational risk factors with the prevalence of self-reported carpal tunnel syndrome in a national survey of the working population. Am J Industr Med. 32, 550-556.

112. Tyson A (1995). Prevention and rehabilitation of shoulder impingement. Strength and Conditioning. 17, 31-33.

113. US Department of Labor (1999). CTD News. http//ctdnews.com/bls.html.

114. Weber W (2000). Dutch health council calls for more RSI research. Lancet 356, 1994.

115. Weinberg RS, Gould D (1995). Foundations of Sport and Exercise Psychology. Champaign: Human Kinetics, 299-316, 91-114.

116. Weinstein SM, Herring SA (1992). Nerve problems and compartment syndromes in the hand, wrist, and forearm. Clin Sports Med. 11, 161-188. 
117. Werner RA, Andary M (2002). Carpal tunnel syndrome: pathophysiology and clinica neurophysiology. Clin Neurophysiol. 113, 1373-1381.

118. Werner RA, Armstrong TJ (1997). Carpal tunnel syndrome: ergonomic risk factors and intra carpal pressure, carpal tunnel syndrome. Phys Med and Rehabil. 8, 555-569.

119. Werner RA, Armstrong TJ, Aylard MK (1997). Intracarpal canal pressure: the role of finger, hand, wrist and forearm position. Clin Biom. 12, 44-51.

120. Williams TL, Smith LA, Herrick RT (1989). Exercise as a prophylactic device against carpal tunnel syndrome. Santa Monica: Human Factor Society.

121. Willis JD, Champbell LF (1992). Exercise Psychology. Champaign: Human Kinetics, 39-78.

122. Wilmore JH, Costill DL (1994). Physiology of Sport and Exercise. Champaign: Human Kinetics, 15-17, 490-511.

123. Wu S-P, Hsieh C-S (2002). Ergonomics study on the handle length and lift angle for the culinary spatula. Appl Ergonomics. 33, 493-501. 